\title{
Dermoscopy of pigmented lesions on mucocutaneous junction and mucous membrane
}

\author{
J. Lin, H. Koga, M. Takata and T. Saida \\ Department of Dermatology, Shinshu University School of Medicine, Asahi 3-1-1, \\ Matsumoto 390-8621, Japan
}

\section{Summary}

Background The dermoscopic features of pigmented lesions on the mucocutaneous junction and mucous membrane are different from those on hairy skin. Differentiation between benign lesions and malignant melanomas of these sites is often difficult.

Objective To define the dermoscopic patterns of lesions on the mucocutaneous junction and mucous membrane, and assess the applicability of standard dermoscopic algorithms to these lesions.

Patients and methods An unselected consecutive series of 40 lesions on the mucocutaneous junction and mucous membrane was studied. All the lesions were imaged using dermoscopy devices, analysed for dermoscopic patterns and scored with algorithms including the ABCD rule, Menzies method, 7-point checklist, 3-point checklist and the CASH algorithm.

Results Benign pigmented lesions of the mucocutaneous junction and mucous membrane frequently presented a dotted-globular pattern (25\%), a homogeneous pattern (25\%), a fish scale-like pattern (18-8\%) and a hyphal pattern (18-8\%), while melanomas of these sites showed a multicomponent pattern $(75 \%)$ and a homogeneous pattern (25\%). The fish scale-like pattern and hyphal pattern were considered to be variants of the ring-like pattern. The sensitivities of the ABCD rule, Menzies method, 7-point checklist, 3-point checklist and CASH algorithm in diagnosing mucosal melanomas were $100 \%, 100 \%, 63 \%, 88 \%$ and $100 \%$; and the specificities were $100 \%$, $94 \%, 100 \%, 94 \%$ and $100 \%$, respectively.

Conclusion The ring-like pattern and its variants (fish scale-like pattern and hyphal pattern) are frequently observed as well as the dotted-globular pattern and homogeneous pattern in mucosal melanotic macules. The algorithms for pigmented lesions on hairy skin also apply to those on the mucocutaneous junction and mucous membrane with high sensitivity and specificity. 
Pigmented lesions on the mucocutaneous junction and mucous membrane cover a wide range of disorders including mucosal melanotic macule, melanocytic naevus, malignant melanoma, and nonmelanocytic lesions, such as Bowenoid papulosis. In recent years, the use of dermoscopy has improved the diagnostic accuracy of pigmented skin lesions to a great extent, and the diagnostic algorithms for pigmented lesions on hairy skin ${ }^{1-5}$ and acral volar skin ${ }^{6}$ have been well defined. However, little is known about the role of dermoscopy in the diagnosis of pigmented lesions on the mucocutaneous junction and mucous membrane, which often pose diagnostic problems. ${ }^{7}$ Reports about such lesions are mainly based on isolated cases or a relatively small series of cases. ${ }^{8-11}$ No studies concerning differential diagnosis between malignant melanomas, especially flat lesions of melanoma, and benign pigmented lesions on the mucocutaneous junction and mucous membrane have been published. Here we report the dermoscopic patterns in 40 lesions on the mucocutaneous junction and mucous membrane in Japanese patients, including malignant melanomas. Furthermore, we evaluate whether the algorithms commonly used for distinguishing malignant melanoma from benign lesions on hairy skin are also applicable to the lesions on the mucocutaneous junction and mucous membrane.

\section{Patients and methods}

\section{Patients}

The study was performed on a consecutive unselected series of 40 pigmented lesions on the mucocutaneous junction and mucous membrane obtained from 37 Japanese patients (16 males and 21 females; median age, 48 years; age range, 13-84 years) collected at the Department of Dermatology, Shinshu University Hospital, Japan, between 1 January 2004 and 31 December 2008. All patients gave written informed consent for dermoscopic imaging and most patients also gave consent for biopsy. For all lesions, the diagnosis was histopathologically confirmed, except for 11 mucosal melanotic macules and two melanocytic nevi that showed no suspicious features under dermoscopy and no changes following consecutive digital monitoring.

\section{Dermoscopic analysis}

Digital dermoscopic images of all the lesions were taken with a Digital Camera System, Derma 9500 (Derma Medical Inc., Yokohama, Japan) combined with a Canon Powershot A620 camera (Canon Inc., Tokyo, Japan). All lesions were observed, dermoscopic features assessed and dermoscopic patterns defined independently by two dermatologists (J.L. and H.K.), who are experts in dermoscopy, and three dermatologists, who are not experts in dermoscopy. All the observers gave a consensus on the dermoscopic patterns of all the lesions. Even those not expert in dermoscopy 
could define these patterns properly. Each lesion was evaluated and scored according to the standard dermoscopic algorithms including the ABCD rule, Menzies method, 7-point checklist, 3-point checklist and CASH algorithm, respectively. ${ }^{1-5}$ A consensus on the presence or absence of a dermoscopic criterion was reached through consultation.

\section{Results}

The 40 lesions investigated included 24 (60\%) mucosal melanotic macules, five (12\%) melanocytic naevi, one ( $2 \%$ ) blue naevus, one (2\%) Bowenoid papulosis, one (2\%) PeutzJeghers syndrome and eight (20\%) malignant melanomas. The sites involved were lip (27 of 40,68\%), penis ( 7 of 40, 18\%), conjunctiva ( 3 of $40,8 \%$ ), vulva ( 2 of $40,5 \%$ ) and anus ( 1 of $40,2 \%$ ) (Table 1). For melanoma lesions, only flat areas on the mucocutaneous junction and mucous membrane were analysed.

Table 1 Clinical characteristics of the 40 lesions

\begin{tabular}{lcccccc}
\hline \multirow{2}{*}{ Diagnosis } & \multicolumn{5}{c}{ Site } & Total \\
\cline { 2 - 6 } & Lip & Penis & Conjunctiva & Vulva & Anus & \\
\hline Melanosis & 20 & 4 & & & & 24 \\
Melanoma & 4 & & 1 & 2 & 1 & 8 \\
Melanocytic nevus & 1 & 2 & 2 & & & 5 \\
Blue nevus & 1 & & & & 1 \\
Bowenoid papulosis & & 1 & & & 1 \\
Peutz-Jegher's & 1 & & & & 1 \\
syndrome & 27 & 7 & 3 & 2 & 1 & 40 \\
\hline Total & & & & & \\
\hline
\end{tabular}

Dermoscopic analysis identified a total of seven patterns in our series of lesions. Collectively, we observed the dottedglobular pattern in eight (20\%) lesions, the homogeneous pattern in $10(25 \%)$ lesions, the multicomponent pattern in six (15\%) lesions, the ring-like pattern in three ( $8 \%$ ) lesions, the fingerprint-like pattern in one (2\%) lesion, and two new patterns, the fish scale-like pattern and the hyphal pattern, in six (15\%) lesions each. The frequency of the seven patterns observed in different diseases on the mucocutaneous junction and mucous membrane is shown in Table 2. The benign lesions mainly presented the dotted-globular pattern ( 8 of $32,25 \%$ ), the homogeneous pattern ( 8 of $32,25 \%$ ), the fish scale-like pattern ( 6 of $32,19 \%$ ), the hyphal pattern ( 6 of $32,19 \%)$, the ring-like pattern ( 3 of $32,9 \%)$ and the fingerprintlike pattern 
(1 of 32, 3\%) (Fig. 1). Malignant melanomas mainly presented with the multicomponent pattern ( 6 of $8,75 \%)$ and the homogeneous pattern ( 2 of $8,25 \%)$.

Table 2. Frequency of dermoscopic patterns compared to different diseases on mucosa and mucocutaneous junction

\begin{tabular}{|c|c|c|c|c|c|c|c|c|}
\hline Diagnosis & $\begin{array}{l}\text { Dotted- } \\
\text { globular }\end{array}$ & Hyphal & $\begin{array}{c}\text { Fish scale- } \\
\text { like } \\
\end{array}$ & Ringlike & Homogeneous & $\begin{array}{c}\text { Fingerprint- } \\
\text { like } \\
\end{array}$ & Multicomponent & Total \\
\hline Mucosal melanotic Macule & 6 & 6 & 5 & 3 & 3 & 1 & & $\mathrm{~N}=24$ \\
\hline Melanocytic naevus & 2 & & & & 3 & & & $\mathrm{~N}=5$ \\
\hline Blue nevus & & & & & 1 & & & $\mathrm{~N}=1$ \\
\hline Bowenoid papulosis & & & & & 1 & & & $\mathrm{~N}=1$ \\
\hline Peutz-Jepher's syndrome & & & 1 & & & & & $\mathrm{~N}=1$ \\
\hline Melanoma & & & & & 2 & & 6 & $\mathrm{~N}=8$ \\
\hline
\end{tabular}



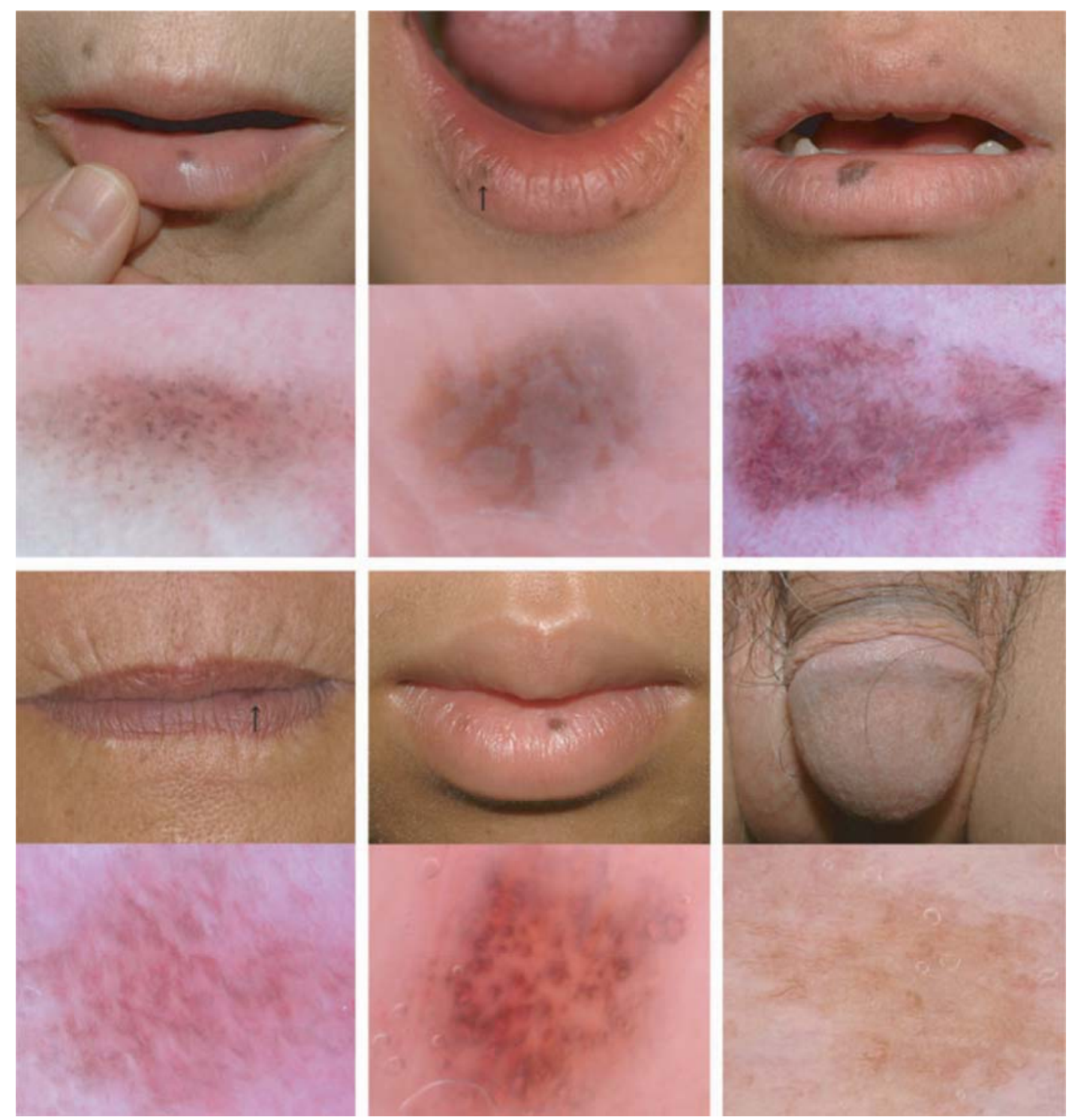

(a) (b) (c)
(d) (e) (f)

Fig 1. Dermoscopic patterns observed in benign pigmented lesions of the mucocutaneous junction and mucous membrane. (a) Macroscopic and dermoscopic photograph of a benign labial melanotic freckle (lentigo) showing dotted-globular pattern. (b) Macroscopic and dermoscopic photograph of a benign labial melanotic freckle (lentigo) ( $\uparrow$ ) showing homogeneous pattern. (c) Macroscopic and dermoscopic photograph of a benign labial melanotic freckle (lentigo) showing fish scale-like pattern. (d) Macroscopic and dermoscopic photograph of a benign labial melanotic freckle (lentigo) ( $\uparrow$ ) showing hyphal pattern. (e) Macroscopic and dermoscopic photograph of a benign labial melanotic freckle (lentigo) showing ring-like pattern. (f) Macroscopic and dermoscopic photograph of a genital lentigo on the penis showing fingerprint-like pattern. 
Although most of these patterns have been reported previously, ${ }^{11,12}$ the fish scale-like pattern and the hyphal pattern, which are frequently observed on mucosal lesions, are described here for the first time. The fish scale-like pattern was observed in five lesions of benign labial melanotic freckle (lentigo) and one lesion of Peutz-Jeghers syndrome, characterized by multiple curves of semicircle, ' $U$ ' or ' $V$ ' shape mimicking the scales of fish. The hyphal pattern was observed in five benign labial melanotic freckles (lentigines) and one genital lentigo on the penis, characterized by less regularly curved or flexed lines of different lengths mimicking fungal hyphae (Fig. 2).

(a)

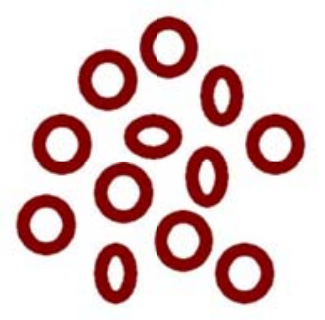

(b)

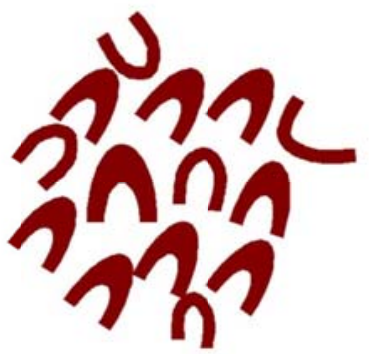

(c)

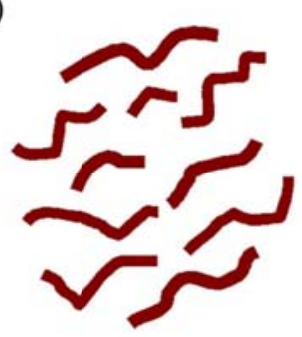

Fig 2. Pattern diagrams of (a) ringlike pattern, (b) fish scale-like pattern and (c) hyphal pattern.

By observing the different dermoscopic images from the same lesion, we consider the fish scale-like pattern to be a variant of the ring-like pattern. In the case of pigmented acral skin lesions, the fibrillar pattern is a variant of the parallel furrow pattern caused by mechanical pressure. ${ }^{13}$ Similarly, the fish scale-like pattern is a variant of the ring-like pattern caused by tension produced when taking the image from different directions. As shown in Figure 3, the same labial melanotic freckle (lentigo) of a 17-year-old male presented with the ring-like pattern in one image, but the fish scale-like pattern in another image. 

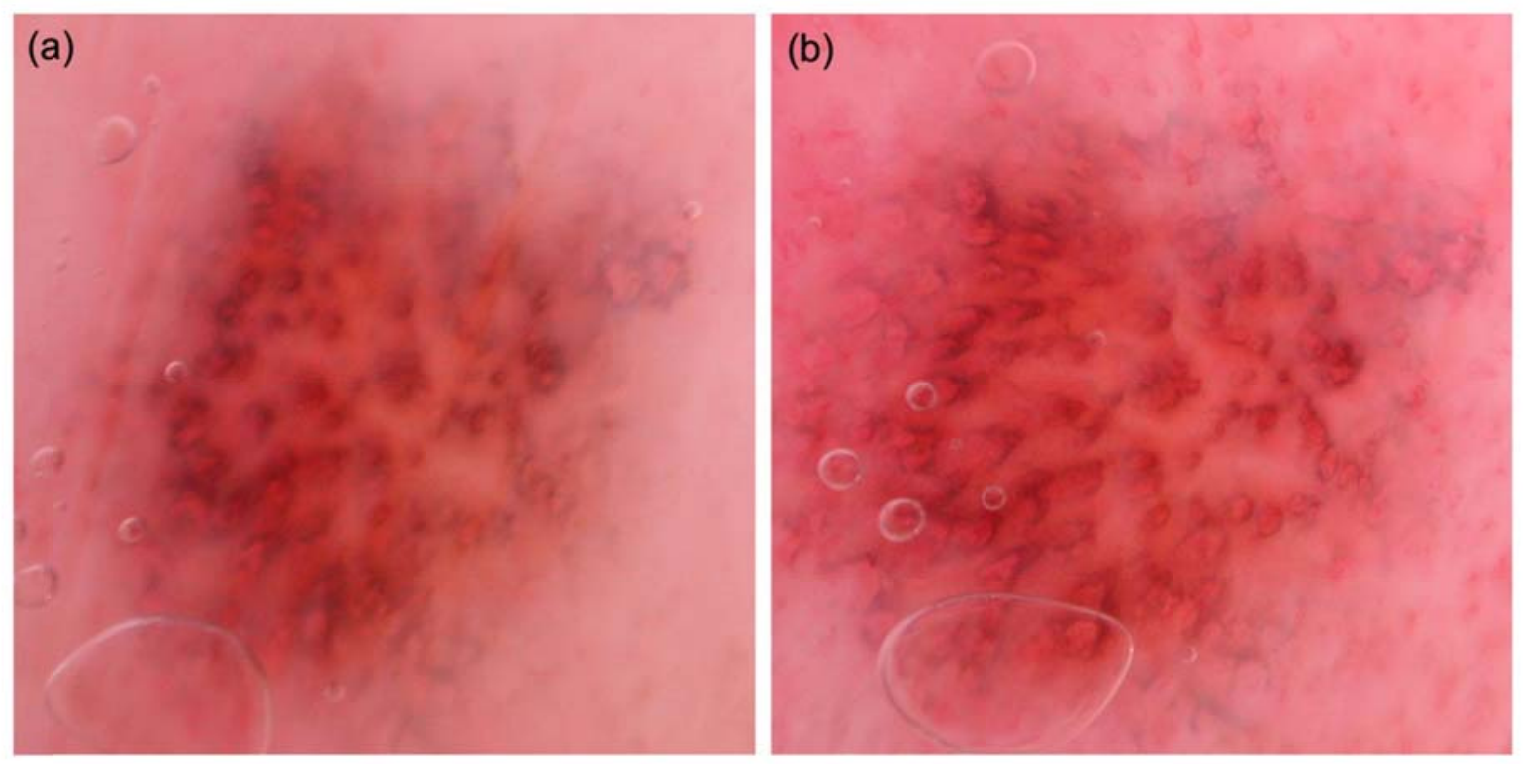

Fig 3. The (a) ringlike pattern and (b) fish scale-like pattern of the same lesion shown by different dermoscopic images of the same lesion.

The most frequent diagnosis in our series of pigmented lesions on the mucocutaneous junction and mucous membrane was mucosal melanotic macule. It includes the benign labial melanotic freckle, labial lentigo and genital lentigo. The 24 mucosal melanotic macules observed in our study include 20 benign labial melanotic freckles (or labial lentigines) and four genital lentigines on the penis. The frequency of the dermoscopic patterns observed in mucosal melanotic macules of these two anatomic sites is shown in Table 3. No obvious correlation between dermoscopic patterns and anatomic sites was observed.

Table 3. Frequency of the dermoscopic patterns observed in melanoses, compared to different sites

\begin{tabular}{lccccccc}
\hline Site & $\begin{array}{c}\text { Dotted- } \\
\text { globular }\end{array}$ & Hyphal & $\begin{array}{c}\text { Fish scale- } \\
\text { like }\end{array}$ & Ringlike & Homogeneous & $\begin{array}{c}\text { Fingerprint- } \\
\text { like }\end{array}$ & Total \\
\hline Lip & 4 & 5 & 5 & 3 & 3 & & $\mathrm{~N}=20$ \\
Penis & 2 & 1 & & & & 1 & $\mathrm{~N}=4$ \\
\hline
\end{tabular}

On histopathological examination, all the mucosal melanotic macules showed a variable increase of melanin pigment along the basal layer and slightly increased melanophages, with no increase in the number of melanocytes (Fig. 4). The fish scale-like pattern, which was considered to be a variant of the ring-like pattern, shared almost the same histopathological findings as the ring-like pattern. The hyphal pattern 
differed from the ring-like pattern and the fish scale-like pattern only in its obliquely elongated rete ridges. Therefore, the hyphal pattern was also considered to be a variant of the ring-like pattern, their histopathological findings being essentially the same. It presents the shape of hyphae just because of the contiguity of the hyperpigmented obliquely elongated rete ridges here and there.

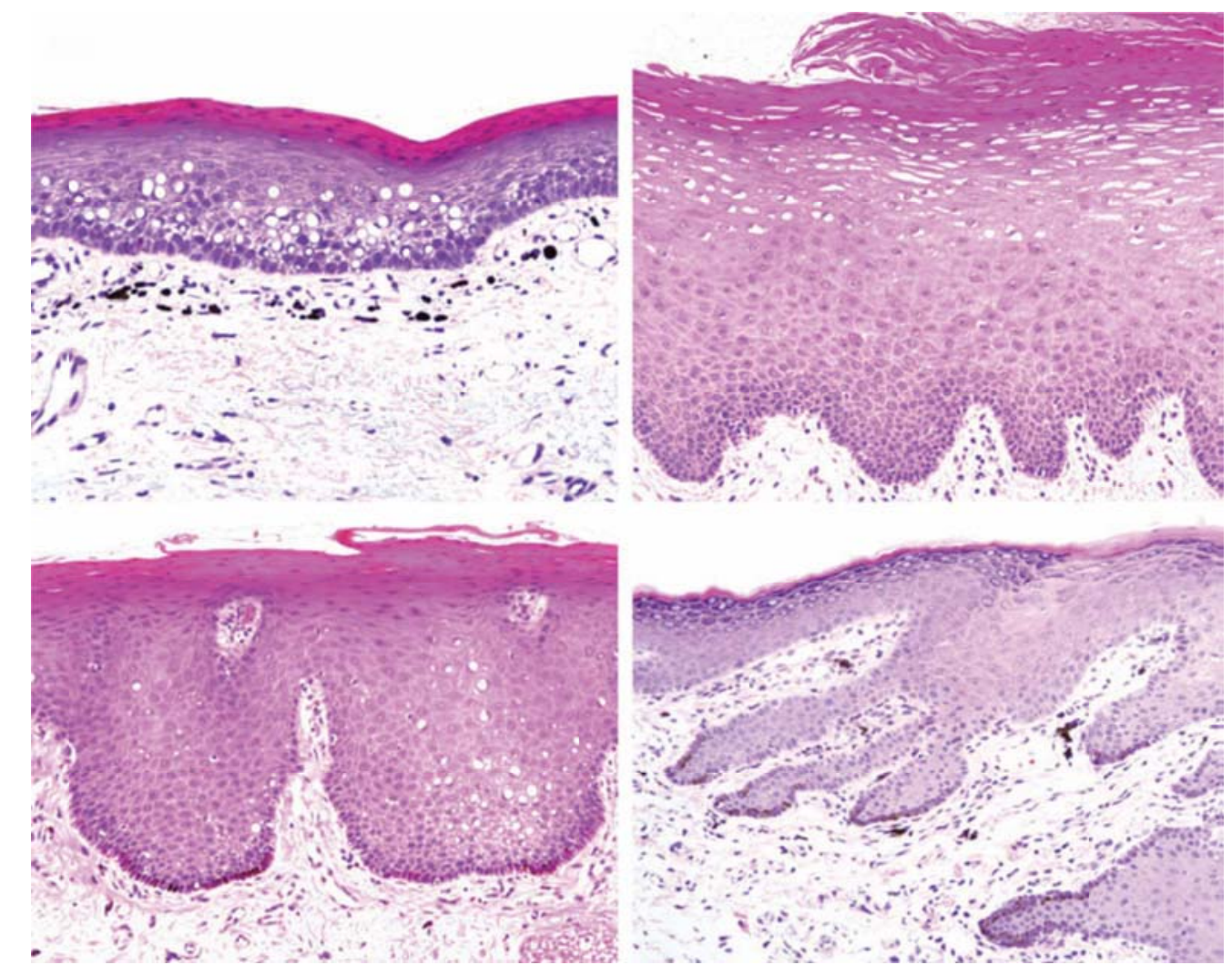

(a) (b)

(c) (d)

Fig 4. Histopathological findings of different patterns in benign labial melanotic freckle (lentigo). (a) The dotted-globular pattern showed locally aggregated melanin in the upper dermis, (b) the homogeneous pattern showed acanthosis and relatively flat rete ridges, (c) the ring-like pattern and the fish scale-like pattern shared the same histopathological characteristics showing hyperpigmented rete ridges with skip areas of pigmentation at the top of the dermal papillae, (d) the hyphal pattern showed hyperpigmentation at the tip of the obliquely elongated rete ridges.

The dermoscopic features observed in the eight malignant melanomas in this study are summarized in Table 4. By observing the dermoscopic features of mucosal 
melanomas compared with those of the benign pigmented lesions, we found that the features most frequently observed in melanomas on the mucocutaneous junction and mucous membrane were asymmetry of structure ( 8 of $8,100 \%)$, multiple colours ( 8 of 8 , $100 \%$ ), blue-white veil (6 of $8,75 \%$ ), irregular dots or globules (5 of $8,62 \%)$, regression structure (3 of $8,38 \%$ ), blotches ( 2 of $8,25 \%)$, irregular vessels ( 2 of $8,25 \%)$ and an irregular pigment network ( 3 of $8,38 \%$ ). Figure 5 shows a typical multicomponent structure of a melanoma on the lip, presenting various dermoscopic features.

Table 4. Dermoscopic features of the 8 lesions of malignant melanoma on mucosa or mucocutaneous junction

\begin{tabular}{lcccccccccc}
\hline \multirow{2}{*}{ Dermoscopic features } & \multicolumn{1}{c}{ Patients } & \multicolumn{1}{c}{ Percent } \\
\cline { 2 - 8 } & $\mathbf{1}$ & $\mathbf{2}$ & $\mathbf{3}$ & $\mathbf{4}$ & $\mathbf{5}$ & $\mathbf{6}$ & $\mathbf{7}$ & $\mathbf{8}$ & $\mathbf{( \% )}$ \\
\hline Anatomical site & Lip & Anus & Vulva & Lip & Conjunctiva & Lip & Lip & Vulva & \\
Asymmetry of structure & $2^{\mathrm{a}}$ & 2 & 2 & 2 & 2 & 2 & 2 & 2 & 100 \\
Multiple colours & $\mathrm{Y}$ & $\mathrm{Y}$ & $\mathrm{Y}$ & $\mathrm{Y}$ & $\mathrm{Y}$ & $\mathrm{Y}$ & $\mathrm{Y}$ & $\mathrm{Y}$ & 100 \\
Homogeneous regions & $\mathrm{N}$ & $\mathrm{Y}$ & $\mathrm{Y}$ & $\mathrm{Y}$ & $\mathrm{Y}$ & $\mathrm{Y}$ & $\mathrm{N}$ & $\mathrm{N}$ & 62 \\
Irregular network & $\mathrm{N}$ & $\mathrm{N}$ & $\mathrm{Y}$ & $\mathrm{Y}$ & $\mathrm{N}$ & $\mathrm{N}$ & $\mathrm{N}$ & $\mathrm{Y}$ & 38 \\
Irregular dots/globules & $\mathrm{Y}$ & $\mathrm{Y}$ & $\mathrm{N}$ & $\mathrm{N}$ & $\mathrm{Y}$ & $\mathrm{N}$ & $\mathrm{Y}$ & $\mathrm{Y}$ & 62 \\
Blotches & $\mathrm{Y}$ & $\mathrm{N}$ & $\mathrm{N}$ & $\mathrm{N}$ & $\mathrm{N}$ & $\mathrm{Y}$ & $\mathrm{N}$ & $\mathrm{N}$ & 25 \\
Blue-white veil & $\mathrm{Y}$ & $\mathrm{Y}$ & $\mathrm{Y}$ & $\mathrm{Y}$ & $\mathrm{N}$ & $\mathrm{Y}$ & $\mathrm{N}$ & $\mathrm{Y}$ & 75 \\
Regression structure & $\mathrm{Y}$ & $\mathrm{Y}$ & $\mathrm{N}$ & $\mathrm{N}$ & $\mathrm{N}$ & $\mathrm{N}$ & $\mathrm{N}$ & $\mathrm{Y}$ & 38 \\
Irregular vessels & $\mathrm{N}$ & $\mathrm{Y}$ & $\mathrm{Y}$ & $\mathrm{N}$ & $\mathrm{N}$ & $\mathrm{N}$ & $\mathrm{Y}$ & $\mathrm{N}$ & 38 \\
Pattern & $\mathrm{M}$ & $\mathrm{M}$ & $\mathrm{M}$ & $\mathrm{M}$ & $\mathrm{H}$ & $\mathrm{H}$ & $\mathrm{M}$ & $\mathrm{M}$ & - \\
\hline
\end{tabular}

${ }^{\mathrm{a}}$ Asymmetry on both axes. $\mathrm{Y}$, observed criterion present; $\mathrm{N}$, criterion absent. M, multicomponent pattern; $\mathrm{H}$, homogeneous pattern.

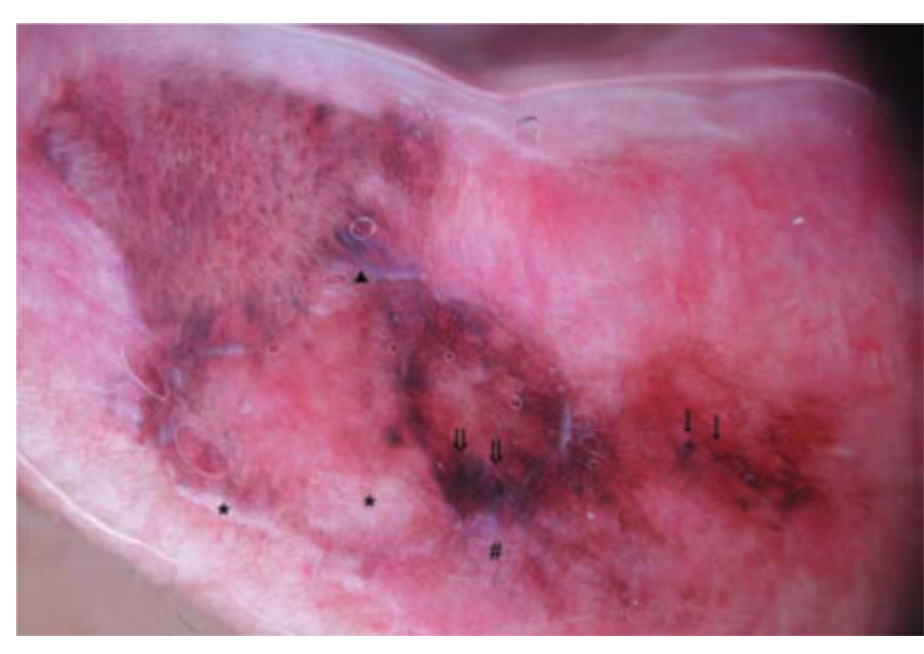

Fig 5. The dermoscopic features of a lesion of malignantmelanoma on the lip (patient 1 in Table 4), showing multicomponent pattern: asymmetry on both axes, four kinds of colours (light brown, dark brown, black and grey-blue), irregular dots (fl), blotches ( $\Downarrow)$, scar-like depigmentation $(*)$ and peppering (\#), and blue-white veil 
Furthermore, all the lesions were evaluated and scored according to the standard dermoscopic algorithms. The sensitivities of the ABCD rule, Menzies method, 7-point checklist, 3-point checklist and CASH algorithm in diagnosing melanomas on the mucocutaneous junction and mucous membrane were 100\% (8 of 8), 100\% (8 of 8), 63\% (5 of 8), 88\% (7 of 8) and 100\% (8 of 8); and the specificities were 100\% (32 of 32), 94\% (30 of 32 ), 100\% (32 of 32), 94\% (30 of 32) and 100\% (32 of 32), respectively. This suggests that the algorithms for lesions on hairy skin also apply to pigmented lesions on the mucocutaneous junction and mucous membrane, with sensitivity and specificity comparable with those on the hairy skin.

\section{Discussion}

In our series of pigmented lesions on the mucocutaneous junction and mucous membrane, mucosal melanotic macule $(60 \%)$ was the most frequent diagnosis. Mucosal melanotic macule is a benign pigmented lesion of mucosa due to hyperpigmentation of basal keratinocytes, with melanocytes normal or slightly increased in number. ${ }^{14,15}$ Although the dermoscopic features of pigmented lesions have been extensively described on glabrous and nonglabrous skin, little is known about those of benign mucosal melanotic macule. Pigment network, brown globules and streaks were thought be dermoscopic features associated with an increased number of melanocytes arranged in nests, ${ }^{16}$ and the structureless pattern lacking such dermoscopic features was considered to be a hallmark of benign mucosal melanotic macule. ${ }^{10}$ Francesca et al. ${ }^{11}$ identified three major dermoscopic patterns in benign mucosal melanotic macule, known as parallel pattern, structureless pattern and reticular-like pattern. Angela et al. ${ }^{12}$ reported a ringlike pattern in a larger series of 87 lesions of vulvar melanoses. In our study of 24 mucosal melanotic macules on the lips and the penis, we observed six different dermoscopic patterns, including dotted-globular pattern, homogeneous pattern, ringlike pattern, fingerprint-like pattern, and two new dermoscopic patterns we proposed and defined here for the first time, the fish scale-like pattern and hyphal pattern.

The dotted-globular pattern was mainly observed in the benign mucosal melanotic macule and melanocytic naevus. However, the histopathological correlates of dots and globules in the mucosal melanotic macule and melanocytic naevus were different. The dots in mucosal melanotic macule were due to aggregated melanin in the upper dermis, whereas the dots and globules in melanocytic naevus correlated histopathologically to aggregated pigmented melanocytes or melanophages. The homogeneous pattern was an alternative expression of the previously reported structureless pattern. ${ }^{11}$ The 
fingerprint-like pattern was similar to the parallel pattern described by Francesca et al. ${ }^{11}$ However, the lines observed in our case of fingerprintlike pattern are thinner and curved, just like the fingerprint. Considering its differences from the parallel pattern of the acral lesions, we prefer the expression of 'fingerprint-like pattern'. The fish scale-like pattern and the hyphal pattern, two new patterns frequently observed in our series of mucosal melanotic macule, were considered to be variants of the ring-like pattern, with essentially the same histopathological findings, characterized by hyperpigmentation of the rete ridges along the basal layer, with the top of dermal papillae less or nonpigmented. These two new patterns as well as the ring-like pattern are quite distinctive patterns of mucosal melanotic macule.

Mucosal malignant melanomas, especially the flat lesions, often pose diagnostic challenges, because at times they share some features with benign mucosal melanotic macule. Mucosal melanomas account for fewer than 5\% of all melanomas, ${ }^{17,18}$ but they tend to be more aggressive than their cutaneous counterparts, developing at sites rich in blood vessels and lymphatic vessels. 19 Thus, it is of paramount importance to make an early diagnosis. Although the use of dermoscopy has advanced greatly in the diagnosis of pigmented skin lesions, the algorithm for differential diagnosis of pigmented mucosal lesions remains undefined. In this study, we scored each lesion using the algorithms applicable to hairy skin, including the ABCD rule, Menzies method, 7-point checklist, 3-point checklist and CASH algorithm. Most of the above dermoscopic algorithms showed high sensitivity (62-100\%) and specificity (94-100\%) in mucosal melanomas, which are comparable with their sensitivity and specificity in hairy skin. The results suggest that the algorithms for pigmented lesions of the hairy skin also apply to lesions on mucocutaneous junction and mucous membrane.

Most of the currently accepted dermoscopic scoring systems involve the number of colours to evaluate pigmented lesions. Different from hairy skin, the contact dermoscopic image of mucosa often has the background colour white, and usually abounds in vessels showing red. Due to these anatomical characteristics, it is of importance to differentiate them from the white colour of regression and the red colour of proliferating vessels frequently observed in melanoma, and not to overrate such background colours in the dermoscopic image of benign mucosal lesions.

In conclusion, we observed a series of pigmented lesions on the mucocutaneous junction and mucous membrane including benign mucosal melanotic macule, melanocytic naevi, and malignant melanomas; and defined two new dermoscopic patterns, the fish scale-like pattern and the hyphal pattern, which are frequently observed in Japanese mucosal melanotic macules. We assessed the mucosal lesions with 
the commonly used algorithms for lesions on hairy skin, and found that the algorithms also apply to lesions on the mucocutaneous junction and mucous membrane, with high sensitivity and specificity in distinguishing malignant melanoma from benign lesions. Altogether, these results throw light on the practical usage of dermoscopy on pigmented lesions of the mucocutaneous junction and mucous membrane. The use of dermoscopy may also help in the proper management of pigmented lesions on the mucocutaneous junction and mucous membrane, providing additional diagnostic information invisible to the naked eye.

\section{References}

1 Scoltz W, Riemann A, Cognetta AB, Pillet L. ABCD rule of dermatoscopy: a new practical method for early recognition of malignant melanoma. Eur J Dermatol 1994; $4: 521-7$.

2 Menzies SW, Ingvar C, Crotty K, McCarthy WH. Frequency and morphologic characteristics of invasive melanomas lacking specific surface microscopic features. Arch Dermatol 1996; 132:1178-82.

3 Argenziano G, Fabbrocini G, Carli P et al. Epiluminescence microscopy for the diagnosis of doubtful melanocytic skin lesions. Comparison of the ABCD rule of dermatoscopy and a new seven-point checklist based on pattern analysis. Arch Dermatol 1998; 134:1563-70.

4 Soyer HP, Argenziano G, Zelaudek I et al. Three-point checklist of dermoscopy. A new screening method for early detection of melanoma. Dermatology 2004; 208:27-31.

5 Henning JS, Dusza SW, Wang SQ et al. The CASH (color, architecture, symmetry, and homogeneity) algorithm for dermoscopy. J Am Acad Dermatol 2007; 56:45-52.

6 Saida T, Koga H. Dermoscopic patterns of acral melanocytic nevi: their variations, changes and significance. Arch Dermotol 2007; 143:1423-6.

7 Sison-Torre EQ, Ackerman AB. Melanosis of the vulva: a clinical simulator of malignant melanoma. Am J Dermatopathol 1985; 7:51-60.

8 Roberto B, Silvano M, Carlo C. Melanoma of the glans penis. Eur J Dermatol 2005; $15: 113-15$.

9 Matsushita S, Kageshita T, Ishihara T. Comparison of dermoscopic and histopathological findings in a mucous melanoma of the lip. $\mathrm{Br} \mathrm{J}$ Dermatol 2005; 152:1324-6.

10 Carli P, De Giorgi V, Cattaneo A, Giannotti B. Mucosal melanosis clinically 
mimicking malignant melanoma: non-invasive analysis by epiluminescence microscopy. Eur J Dermatol 1996; 6:434-6.

11 Francesca M, Vincenzo DG, Antonio C et al. Dermoscopic features of mucosal melanosis. Dermatol Surg 2004; 30:1118-23.

12 Angela F, Pierluigi B, Renato $\mathrm{C}$ et al. The ringlike pattern in vulvar melanosis. Arch Dermatol 2008; 144:1030-4.

13 Miyazaki A, Saida T, Koga H et al. Anatomical and histopathological correlates of the dermoscopic patterns seen in melanocytic nevi on the sole: a retrospective study. J Am Acad Dermatol 2005; 53:230-6.

14 Barnhill RL, Albert LS, Shama SK et al. Genital lentiginosis: a clinical and histopathologic study. J Am Acad Dermatol 1990; 22:453-60.

15 Kenneth K, Ho L, Dervan P et al. Labial melanotic maculae: a clinical histopathologic and ultrastructural study. J Am Acad Dermatol 1993; 28:33-9.

16 Yadav S, Vossaert KA, Kopf AW et al. Histopathological correlates of structures seen on dermoscopy (epiluminescence microscopy). Am J Dermatopathol 1993; 15:297-305.

17 Wick MR. Prognostic factors for cutaneous melanoma. Am J Clin Pathol 1998; 110:713-18.

18 Saida T, Kawachi S, Takata M et al. Histopathologic characteristics of malignant melanoma affecting mucous membranes: a unifying concept of histogenesis. Pathology 2004; 36:404-13.

19 Froix AJ, Salti GI. Primary malignant melanoma of the lip. J Surg Oncol 2003; 84:79 . 